\title{
The extended structure of the remote cluster B514 in M 31
}

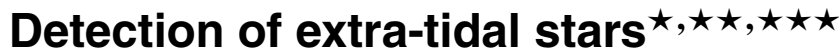

\author{
L. Federici, M. Bellazzini, S. Galleti, F. Fusi Pecci, A. Buzzoni, and G. Parmeggiani
}

\begin{abstract}
INAF - Osservatorio Astronomico di Bologna, via Ranzani 1, 40127 Bologna, Italy
e-mail: [luciana.federici;michele.bellazzini ; flavio. fusipecci; alberto.buzzoni ; gianluigi.parmeggiani]@oabo.inaf.it; silvia.galleti2@unibo.it
\end{abstract}

Received 18 January 2007 / Accepted 6 June 2007

\section{ABSTRACT}

\begin{abstract}
Aims. We present a study of the density profile of the remote M 31 globular cluster B514, obtained from HST/ACS observations. Methods. By coupling analysis of the distribution of the integrated light with star counts, we are able to reliably follow the profile of the cluster out to $r \sim 35^{\prime \prime}$, corresponding to $\simeq 130 \mathrm{pc}$. The profile is well-fitted out to $\sim 15$ core radii by a King Model having $C=1.65$. With an estimated core radius $r_{\mathrm{c}}=0.38^{\prime \prime}$, this corresponds to a tidal radius of $r_{\mathrm{t}} \sim 17^{\prime \prime}(\sim 65 \mathrm{pc})$. The analysis of the light profile also allows an estimate of the ellipticity and position angle of the isophotes within $r \leq 20^{\prime \prime}$.

Results. We find that both the light and the star-count profiles show a departure from the best-fit King model for $r \gtrsim 8^{\prime \prime}-$ as a surface brightness excess at large radii, and the star-count profile shows a clear break in the correspondence of the estimated tidal radius. Both features are interpreted as the signature of the presence of extra tidal stars around the cluster. It is also shown that B514 has a significantly larger half-light radius than ordinary globular clusters of the same luminosity. In the $M_{V}$ vs. $\log r_{\mathrm{h}}$ plane, B514 lies in a region inhabited by peculiar clusters, like $\omega$ Cen, G1, NGC 2419 and others, as well as by the nuclei of dwarf elliptical galaxies.
\end{abstract}

Key words. galaxies: individual: M 31 - galaxies: star clusters - catalogs - galaxies: local group - stellar dynamics

\section{Introduction}

Until a couple of years ago we have lacked any knowledge of M 31 clusters at large (projected) distances from the center of the galaxy; the farthest known cluster was $\mathrm{G} 1$, at $R_{\mathrm{p}} \sim 35 \mathrm{kpc}$ from the center of M 31, while (a few) Galactic globulars are found out to a galactocentric distance of $R_{\mathrm{GC}} \sim 120 \mathrm{kpc}$ (10 at $R_{\mathrm{GC}}>30 \mathrm{kpc}$, according to Harris 1996). Recent searches have identified several new remote clusters ${ }^{1}$ in M 31 (Huxor et al. 2004; Galleti et al. 2005; Bates et al. 2004; Martin et al. 2006). These studies seem to indicate that a relatively large number of globulars are still to be discovered in the extreme outskirts of M 31. A significant sample of distant globulars may provide extremely useful information on the early evolution of the M 31 halo. In particular, it has been suggested that bright globular clusters may be the remnants of disrupted nucleated dwarf galaxies (Freeman \& Bland-Hawthorn 2002, and references therein; see also Brodie \& Strader 2006). If this were the case, the probability of finding the observational fingerprints of these kinds of phenomena is much higher at large distances from the center of

* Based on observations with the NASA/ESA Hubble Space Telescope obtained at the Space Telescope Science Institute (STScI), which is operated by AURA, Inc., under NASA contract NAS 5-26555.

$\star \star$ Table 5 is only available in electronic form at the CDS via anonymous ftp to cdsarc.u-strasbg.fr (130.79.128.5) or via http://cdsweb.u-strasbg.fr/cgi-bin/qcat?]/A+A/473/429

$\star \star \star$ Tables 3 and 4 are only available in electronic form at http://www . aanda.org

${ }^{1}$ In the present context we will call remote clusters those lying at galactocentric distances larger than $30 \mathrm{kpc}$. parent galaxies, where substructures may survive for long times (Bullock \& Johnston 2004) and the overall stellar density is very low. Moreover, the structure and evolution of clusters orbiting in very low-density environments is a very interesting topic in itself.

In Galleti et al. (2006b, hereafter G06b) we have presented deep photometry with the Hubble Space Telescope - Advanced Camera for Survey of the recently discovered cluster B514 (Galleti et al. 2005, hereafter G05), lying at $R_{\mathrm{p}} \simeq 55 \mathrm{kpc}$ from the center of M 31. The derived color magnitude diagram (CMD) revealed that B514 is a genuine old and metal-poor globular cluster $([\mathrm{Fe} / \mathrm{H}]=-1.8$, also confirmed by the spectroscopic estimate by G05). The cluster is very bright $\left(M_{V} \simeq-9.1\right)$ and appears quite extended, similar to the brightest remote cluster of the Milky Way, i.e. NGC 2419. Here we present analysis of the surface brightness distribution of B514 obtained from the same HST-ACS data. By coupling the surface brightness profile obtained from the integrated light - for the inner parts - to star counts in the outer region, and thanks to the extremely low level of background density in the field, we were able to identify an unequivocal break in the outer profile of the cluster, indicating the presence of extra-tidal stars (see Johnston et al. 1999; Combes et al. 1999; Grillmair et al. 1995, 1996; Leon et al. 2000).

Extra-tidal components and/or extended tidal tails have been observed in several Galactic globulars (see Grillmair et al. 1995; Leon et al. 2000; Testa et al. 2000; Odenkirchen et al. 2003; Lee et al. 2004; Belokurov et al. 2006, and references therein). Holland et al. (1997) and Barmby et al. (2002, hereafter BHH), 
found some M 31 clusters whose light profile exceeds the bestfit King model $(1962,1966)$ in the outermost regions and interpreted this discrepancy as an extra-tidal component. Grillmair et al. (1996) found the same kind of discrepancy in three M 31 clusters; they were able to follow the density profile of the clusters to significantly below the background level by coupling the light profile with the profile obtained from star-counts. By applying a similar technique, we are able to follow the profile of B514 out to $r \sim 35^{\prime \prime}$. Moreover, we find that B514 has a halflight radius $\left(r_{\mathrm{h}}\right)$ that is larger (by $\gtrsim 15 \%$ ) than typical globular clusters of the same luminosity, a characteristic shared by a few very peculiar systems, like $\omega$ Cen, M54, G1, and NGC 2419 (see Mackey \& van den Bergh 2005, hereafter MB05; and Hasegan et al. 2005, for a thorough discussion).

\section{The surface brightness profile of B514}

Since the details of the observations and data reduction are reported in G06b, we recall just a few essential elements here, and refer the interested reader to that paper. The cluster was observed with the Wide Field Channel (WFC) of the ACS, which has a total field of view of $202^{\prime \prime} \times 202^{\prime \prime}$ and a pixel scale of $0.049^{\prime \prime} \mathrm{pixel}^{-1}$. The observational material is constituted by three $F 606 \mathrm{~W}$ images (total $t_{\exp }=2412 \mathrm{~s}$ ) and three $F 814 \mathrm{~W}$ images (total $t_{\exp }=2419 \mathrm{~s}$ ), as well as the associated combined (drizzled) images. The cluster is placed in the center of one of the two ACS/WFC chips (Chip 2), while Chip 1 samples the field population (see G06b and below). If not otherwise stated, magnitudes are always on the VEGAMAG scale as defined by Sirianni et al. (2005). The reddening corrections are performed as in G06b, assuming $E(B-V)=0.10$; a distance modulus $(m-M)_{0}=24.47$ is also assumed, after McConnachie et al. (2005), corresponding to $D_{\odot}=783 \mathrm{kpc}$. At this distance $1^{\prime \prime}$ correspond to $3.8 \mathrm{pc}$ and one ACS/WFC pixel to $0.19 \mathrm{pc}$.

The extreme crowding conditions prevent full resolution into stars in the densest region of M 31 globular clusters, even at the exquisite spatial resolution achieved by HST cameras. However, very accurate and well-resolved surface brightness profiles can be obtained by studying the distribution of their integrated light (see, for example, Fusi Pecci et al. 1994; Djorgovski et al. 2003; $\mathrm{BHH})$. Although very successful in the bright inner regions of the clusters, this technique is limited in the outermost parts of the clusters, where the low-luminosity density coming from cluster stars may be overwhelmed by the brightness of the background. In this regime, star counts may be much more efficient, since cluster stars may be easily identified out to large radii, under favorable conditions (see Grillmair et al. 1996). The ACS field studied here lies more than three degrees apart from the center of M 31, and it appears to have an exceedingly low density of background stars (see G06b and below). This allowed us to derive a reliable light profile, completely unaffected by incompleteness, out to $r \sim 20^{\prime \prime}$, and to extend the analysis out to $r \sim 35^{\prime \prime}$, in completely uncrowded regions, by counting stars with the colors and magnitudes typical of the cluster population.

\subsection{The light profile}

The light profile has been obtained independently from the $F 606 W$ and $F 814 W$ drizzled images. A few heavily saturated foreground stars (all at $r>26^{\prime \prime}$ from the cluster center) have been excised from the images and replaced with the mean value of the surrounding background, to avoid contaminating the profile.
The light profiles were derived using the XVISTA software maintained by J. Holtzman ${ }^{2}$. XVISTA iteratively resolves the profile by fitting ellipses to the observed light distribution until a stable solution is reached, see Fusi Pecci et al. (1994), Djorgovski et al. (2003), for examples of applications to M 31 clusters, and Lauer (1985), for a detailed description of the use. As output the code provides the coordinates of the center, the surface brightness, the ellipticity $(\epsilon=1 .-b / a)$, and the position angle (PA, in degrees, measured anti-clockwise from the North direction) of each fitted ellipse, as well as the total amount of light enclosed within each ellipse. The profiles are derived with a single pixel step. This resolution is appropriate for the innermost regions of the cluster $\left(r \approx 2^{\prime \prime}-3^{\prime \prime}\right)$ where the light intensity is very high, while provides quite a noisy profile at larger radii. This problem is solved by taking an average of the whole profile over $10 \mathrm{px}$ bins, which sampling ensures a satisfying level of noise out to $r \sim 20^{\prime \prime}$. The background level is estimated as the average surface brightness in large $(\sim 100 \times$ $100 \mathrm{px}^{2}$ ) "empty" areas far away from the cluster. The level of background is very low $\left(\mu_{F 606 W} \sim 29.5 \mathrm{mag} / \operatorname{arcsec}^{2}\right)$, and the final background-subtracted profiles were verified to be very robust to variations of the adopted background. The uncertainty on the position of the center is $\sim 2$ px in the $X$ and $Y$ directions: variations in the assumed position of this size doesn't affect significantly the derived profiles. The derived intensity profiles can be directly converted into magnitude/ $\operatorname{arcsec}^{-2}$ units using the VEGAMAG zero points of Sirianni et al. (2005). The derived $F 660 W$ and $F 814 W$ profiles are shown in Fig. 1 . The profiles are quite smooth, well-behaved, and very similar in shape, at least out to $r \simeq 8^{\prime \prime}$, i.e. the radius enclosing $\simeq 90 \%$ of the whole cluster light. Outside this radius both profiles show a marginal excess of light with respect to the best-fitting King (1962) model (see below), but the $F 814 \mathrm{~W}$ profile appears noisier, probably because of the greater weight associated to the contribution of individual bright RGB stars in this redder passband. The $F 606 \mathrm{~W}$ light profile is reported in Table 3, only available in the electronic edition of the Journal. The apparent integrated magnitudes in the VEGAMAG system $\operatorname{mag}_{t, F 606 \mathrm{~W}}$ and $\operatorname{mag}_{t, F 814 \mathrm{~W}}$ were estimated by integrating the respective light profiles.

We take the half width at half maximum (HWHM) of the profile as the core radius (King 1962; Spitzer 1987). Once this parameter was fixed, we searched for the King's models providing the best fit to both profiles. To take proper account of the ACS point spread function (PSF) in measuring the light profile, the King models were convolved with analytic F606W/F814W PSFs models, modeled on observed bright stars, as done by Barmby et al. (2007). All the comparisons between observed profiles and King's models presented in the following only involve PSF-convolved theoretical profiles. In Table 1 we report both the observed and the de-convolved best-fit parameters (see below). The former must be adopted when dealing with the observed profiles, while the latter must be used in the comparisons with other clusters. We note that the adoption of PSF-convolved profiles results in small changes $(\lesssim 10 \%)$ in the cluster parameters, as usually occurs for extended M 31 clusters like B514 (see Barmby et al. 2007).

The derived $F 660 W$ and $F 814 W$ profiles are shown in Fig. 1, compared to PSF-convolved King's models with concentration parameter $C=\log \left(r_{\mathrm{t}} / r_{\mathrm{c}}\right)=1.4,1.5,1.6,1.7$. If we limit our

\footnotetext{
2 See http://astronomy.nmsu.edu/holtz/xvista/index.html for download and documentation.
} 
Table 1. Observed and de-convolved parameters.

\begin{tabular}{lcccc}
\hline \hline & & Observed & & De-convolved \\
Parameter & $F 606 W$ & $F 814 W$ & Average & Average \\
\hline$r_{\mathrm{c}}[\operatorname{arcsec}]$ & 0.38 & 0.45 & $0.42 \pm 0.03$ & 0.38 \\
$r_{\mathrm{h}}[\operatorname{arcsec}]$ & 1.52 & 1.73 & $1.6 \pm 0.2$ & 1.44 \\
$r_{\mathrm{t}}[\operatorname{arcsec}]$ & 19.0 & 18.0 & $18.8 \pm 2.5$ & 17.0 \\
$\mathrm{C}$ & 1.7 & 1.6 & 1.65 & 1.65 \\
$\mu(0)\left[\mathrm{mag} / \operatorname{arcsec}^{2}\right]$ & 16.41 & 15.83 & & $16.33 / 15.74^{a}$ \\
$\mu_{\mathrm{r}_{\mathrm{h}}}\left[\mathrm{mag} / \operatorname{arcsec}^{2}\right]$ & 18.5 & 17.9 & & $18.4 / 17.5^{b}$ \\
mag $_{\mathrm{t}}[V E G A M A G]$ & $15.48 \pm 0.06$ & $14.71 \pm 0.06$ & & \\
\hline
\end{tabular}

${ }^{a}$ De-convolved $F 606 W / F 814 W$ central surface brightnesses. ${ }^{b}$ De-convolved $F 606 W / F 814 W$ half-light radius surface brightnesses.

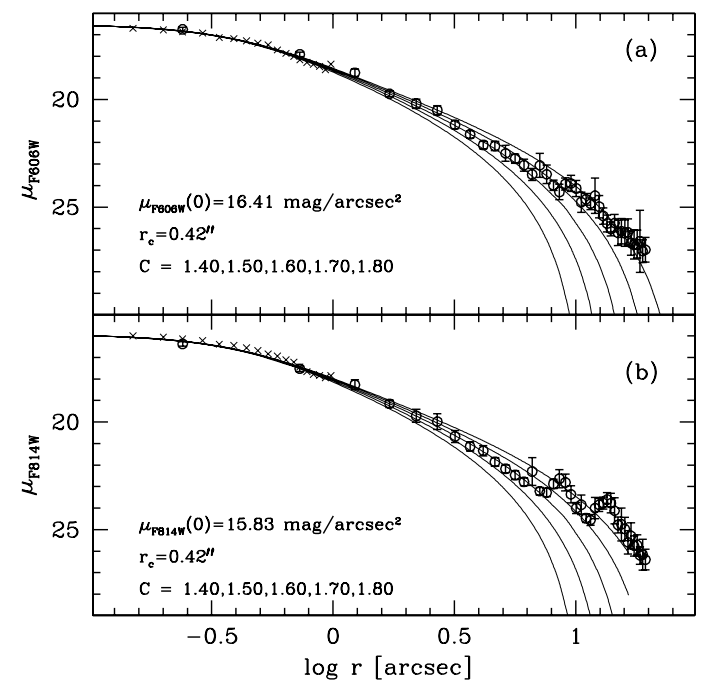

Fig. 1. Surface brightness profiles (in mag/arcsec ${ }^{2}$ ) of B514 in $F 606 \mathrm{~W}$ (upper panel) and $F 814 W$ (lower panel). The "x"s in the 2 " innermost region are from the original 1-pixel step profile, while the open circles with error bars are average values over $10 \mathrm{px}$ bins. The curves are PSFconvolved King models of different concentration, from $C=1.4$ to $C=1.7$, from left to right. The adopted core radius and central surface brightness are also reported.

analysis to the most reliable region within $r=8^{\prime \prime}$ (encompassing $\simeq 19$ core radii), the best fit is achieved with the $C=1.7$ model for the $F 606 W$ profile and $C=1.6$ for the $F 814 W$ profile.

We can gain some insight into the uncertainty associated with many observed/derived parameters by the comparison between the estimates obtained in the $F 606 \mathrm{~W}$ and $F 814 \mathrm{~W}$ profiles. For size parameters, (core radius $r_{\mathrm{c}}$, half-light radius $r_{\mathrm{h}}$, tidal radius $r_{\mathrm{t}}$, see King 1962), we adopt the mean of the independent estimates obtained by the two profiles. For example, for the core radius we obtain $r_{\mathrm{c}}=0.38^{\prime \prime}$ from $F 606 \mathrm{~W}$ and $r_{\mathrm{c}}=0.45^{\prime \prime}$ from $F 814 W$, and we adopt $r_{\mathrm{c}}=0.42 \pm 0.03^{\prime \prime}$. Adopting $C=1.65 \mathrm{we}$ obtain a tidal radius of $r_{\mathrm{t}} \simeq 18.8 \pm 2.5^{\prime \prime}$; for the half-light radius we obtain $r_{\mathrm{h}}=1.52^{\prime \prime}$ from $F 606 W$ and $r_{\mathrm{h}}=1.73^{\prime \prime}$ from $F 814 W$, and we adopt $r_{\mathrm{h}}=1.6 \pm 0.2^{\prime \prime}$ (observed values, see Table 1 ).

The half-light radii were also computed by performing aperture photometry on circular concentric annuli. This independent procedure provided the same results as obtained with XVISTA, indicating that the estimate of this parameter is very robust. Also the estimates of the apparent integrated magnitudes were checked in this way, and the results obtained with different methods are fully consistent.

A summary of the measured structural parameters is presented in Table 1, while Table 2 shows the derived parameters, i.e. those involving assumptions on distance and reddening
Table 2. Derived parameters.

\begin{tabular}{lc}
\hline \hline Parameter & Average/Adopted \\
\hline$M_{V}$ & $-9.1 \pm 0.1$ \\
$M_{I}$ & $-9.9 \pm 0.1$ \\
$\mu_{V}(0)\left[\mathrm{mag} / \mathrm{arcsec}^{2}\right]$ & $16.5^{a}$ \\
$\mu_{I}(0)\left[\mathrm{mag} / \mathrm{arcsec}^{2}\right]$ & $15.7^{a}$ \\
$\mu_{V, r_{\mathrm{h}}}\left[\mathrm{mag} / \operatorname{arcsec}^{2}\right]$ & $18.4^{a}$ \\
$\mu_{I, r_{\mathrm{h}}}\left[\mathrm{mag} / \mathrm{arcsec}^{2}\right]$ & $17.5^{a}$ \\
$r_{\mathrm{c}}[\mathrm{pc}]$ & $1.4^{a}$ \\
$r_{\mathrm{h}}[\mathrm{pc}]$ & $5.4^{a}$ \\
$\langle$ Ellipticity & $0.19 \pm 0.07$ \\
$\langle$ Position Angle $\rangle[\mathrm{deg}]$ & $28 \pm 14$ \\
\hline
\end{tabular}

${ }^{a}$ De-convolved quantities.

and/or transformations to the standard Johnson-Kron-Cousins photometric system. The latter are achieved with the transformations presented in G06b. Note that if Sirianni et al.'s transformations are used instead, slightly brighter $V$ magnitudes are obtained (by $\sim 0.06 \mathrm{mag}$ ), while the final $I$ magnitudes are the same to within $\pm 0.02 \mathrm{mag}$. The $r_{\mathrm{c}}, r_{\mathrm{t}}$ and surface brightness measures reported in Table 2 are derived from average de-convolved values, $\mu_{r_{\mathrm{h}}}$ is the mean surface brightness within $r_{\mathrm{h}}$, while $\mu(0)$ is the central value of the surface brightness.

In a very recent paper, Mackey et al. (2007) reported on the CMDs and (observed) half-light radii and integrated magnitudes of ten M 31 clusters, including B514 (their GC4), from independent ACS observations (in the following we will refer to these clusters as M-GC1, M-GC2, ..., M-GC10). The parameters of B514 obtained by this team are in good agreement with those presented by us here and in Galleti et al. (2006b).

\subsubsection{Color profile, ellipticity, and position angle}

In the upper panel of Fig. 2 we show the color profile of B514. The profile is remarkably constant in the inner 6 ", while it becomes very noisy in the outer regions. It is clear that, in the low-surface-brightness outer parts, the contribution of individual RGB/BHB stars may be important for establishing the local color. A general tendency toward redder colors in the outer regions can be noted, a phenomenon that has also been observed in other clusters (see Djorgovski et al. 1991; Djorgovski \& Piotto 1993; and Barmby et al. 2002). However, in the present case, we regard this trend as marginally significant, given the uncertainties.

The middle and lower panels of Fig. 2 display the ellipticity and position-angle profiles, respectively. In both cases, once it has been verified that the profiles obtained from the $F 606 \mathrm{~W}$ and $F 814 W$ were fully consistent, we averaged the two. B514 


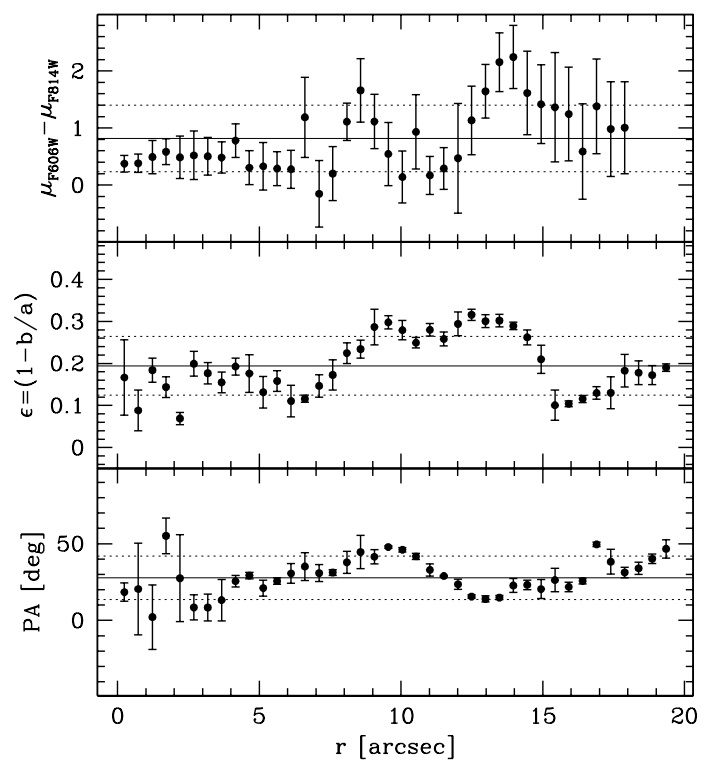

Fig. 2. Upper panel: color profile of B514; the continuous line is the overall mean color and the dotted lines enclose the \pm 1 standard deviation range. Middle panel: ellipticity profile. Lower panel: position angle profile. The last two profiles are the average of the profiles obtained from the $F 606 \mathrm{~W}$ and $F 814 \mathrm{~W}$ images. The meaning of the lines is the same as in the upper panel.

has a mean ellipticity $\langle\epsilon\rangle=0.19 \pm 0.07$, quite high for a globular cluster, but not extraordinary (MB05). A sizable enhancement of the ellipticity is apparent between $r \sim 8^{\prime \prime}$ and $r \sim 15^{\prime \prime}$. A twist of the isophotes seems to occur in the same radial range (lower panel, PA changing by $\sim 30^{\circ}$ ), suggesting a disturbed morphology in this range. The overall conclusion is that the cluster is rather elongated in the NW-SE direction, as is apparent by simply looking at the image (G06b).

\subsection{The profile from star counts}

For star counts we adopt the same catalog as G06b, including only well-measured stars (see G06b, for details). Table 5, available at the CDS, reports the photometry of the individual stars. In the upper panels of Fig. 3 we show the CMDs for the chip containing the cluster (Chip 2) and for the chip presumably sampling the field population (Chip 1, see G06b). The reported contour is the filter we adopt to select likely cluster members on the CMD. It encloses red giant branch (RGB) and horizontal branch (HB) stars having $F 814 W \leq 25.5$. The filter efficiently excludes obvious color outliers and faint stars whose membership can be uncertain. In the lower panels the $X, Y$ map of the two samples - in their relative positions - is presented. The larger circle has a radius of $50^{\prime \prime}$ and is the largest circle that can be fully enclosed within one WFC chip. The following analysis is restricted to filter-selected stars within this circle. The background level of the stellar density is estimated from the whole $50^{\prime \prime}$ circle in Chip 1 as $\rho_{\text {bkg }}=0.0043 \pm 0.0013$ stars $/ \operatorname{arcsec}^{2}$, while we derived the cluster profile from selected stars in Chip 2. It cannot be excluded that cluster stars are also present in Chip 1. However, estimating the background level in the $X<50^{\prime \prime}, X>150^{\prime \prime}$ regions of both chips (lower right panel of Fig. 3) and in the $Y<-50^{\prime \prime}$, $Y>-50^{\prime \prime}$ regions of Chip 1 (separated by the long dashed horizontal line in the lower right panel of Fig. 3), we found that the background is the same as is measured in the $50^{\prime \prime}$ circle, within
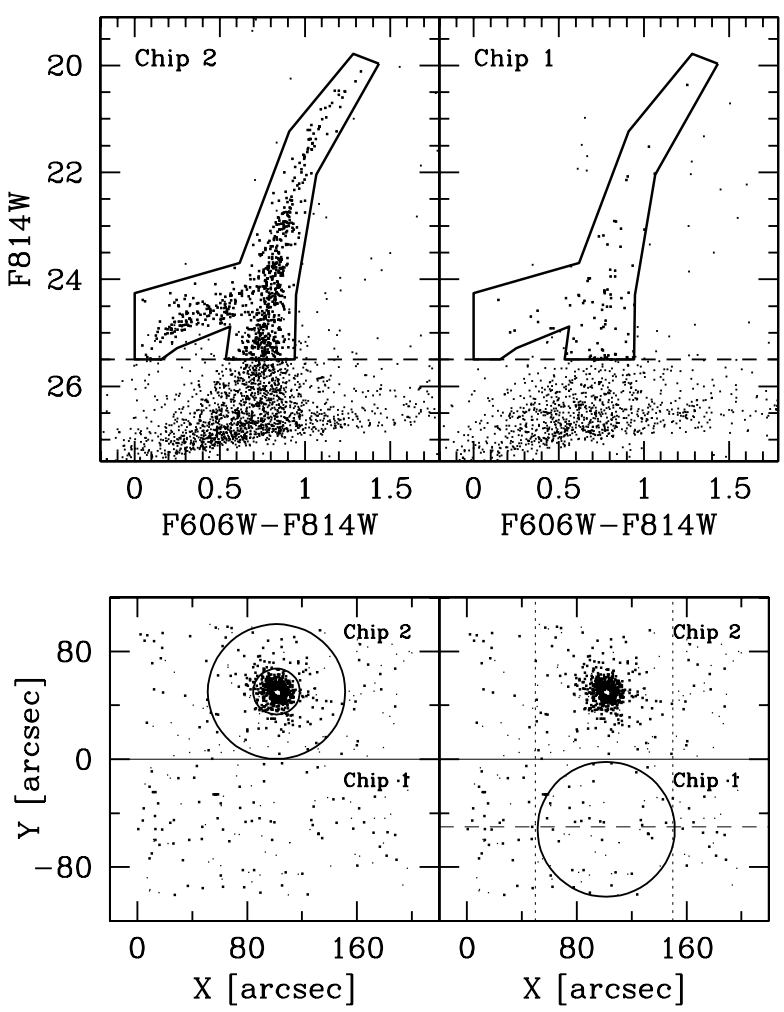

Fig. 3. Selection of likely cluster members on the CMD. Upper panels: CMDs of the stars in the ACS/WFC chip containing the cluster (left panel) and of those in the "empty" chip (right panel). The contour is the filter adopted to select a star for further analysis; the dashed line marks the adopted magnitude limit $(F 814 W<25.5)$. Lower panels: maps of the selected (heavy dots) and unselected (but brighter than $F 814 \mathrm{~W}=$ 25.5) stars of Chip 2 (above the continuous horizontal line) and Chip 1 (below the continuous horizontal line). Here we have adopted a representation that approximately displays the actual relative position of the two chips. The regions selected for the analysis are enclosed within the large circles (radius of 50"). This is the largest circle that can be enclosed in a single chip. The smaller circle in the lower-left panel has $r=20^{\prime \prime}$ and is indicative of the derived tidal limit of the cluster. The dotted vertical lines and the horizontal dashed line plotted in the lower right panel show the portions of the field that have been used to obtain estimates of the background level, which were compared to that obtained in the 50" circle in Chip 1 (see text).

$<2-\sigma$, ranging from $0.0024 \pm 0.0010 \mathrm{stars} / \operatorname{arcsec}^{2}$ to $0.0056 \pm$ $0.0015 \mathrm{stars} / \operatorname{arcsec}^{2}$, and there is no discernible density gradient outside $r=50^{\prime \prime}$ from the cluster center. To have a more reliable estimate of the background level we would need observations of a larger (or more distant) field that, unfortunately, is lacking. However, this implies that, if anything, we are slightly overestimating the background. The possible, associated bias would act against the detection of feeble extra-tidal components, hence it cannot be at the origin of the excess of surface brightness at large radii that is discussed below.

The derived profile is shown in Fig. 4a. The profile is very extended: a level of 5- $\sigma$ above the background is reached at $r \simeq$ $31^{\prime \prime}$, while the (adopted) background level is reached at $r \gtrsim 35^{\prime \prime}$. This plot shows one of the main results of the present paper. Not only do the observed profile clearly extend much beyond the tidal radius derived from the light profiles but, above all, $a$ clear change of slope is detected at $r \sim 18^{\prime \prime}$, i.e. near $r_{\mathrm{t}}$ itself. Note that the excess between $r \simeq 18^{\prime \prime}$ and $r \sim 30^{\prime \prime}$ is many $\sigma$ above the background, making it very significant, even if it encloses just a tiny fraction of the total cluster light. 

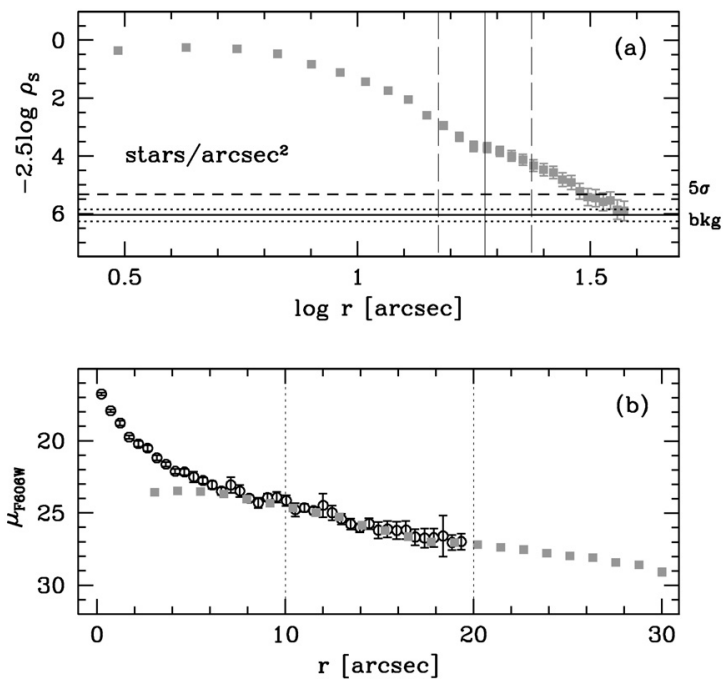

Fig. 4. Panel a): observed surface density profile obtained from star counts (grey squares). The continuous horizontal line marks the background level, the dotted lines mark the $\pm 1-\sigma$ levels around the background, and the dashed line marks the $5-\sigma$ level above the background. The continuous vertical line marks the position of the tidal radius as derived from the fit of the light profile, assuming $C=1.6$, the long-dashed lines show the position of $r_{\mathrm{t}}$ under the assumptions $C=1.55$ (left) and $C=1.75$ (right). Panel b): comparison and match between the $F 606 \mathrm{~W}$ light profile (empty circles) and the (background-subtracted) star counts profile (grey squares). The scale of the horizontal axis has been kept linear to provide a clearer view of the superposition between the two profiles. The dotted lines encloses the region in which the match between the two profile is excellent.

Given the extremely crowded conditions in the inner part of the cluster and the strong density gradient, it is expected that the completeness of the sample is subject to radial variations. Figure $4 \mathrm{~b}$ clearly illustrates the actual case by comparing the light profile (which is completely unaffected by incompleteness) and the background-subtracted star counts profile (reported in Table 4, only available in the electronic edition of the Journal). A linear radial scale is adopted to provide a clearer comparison. This plot shows that incompleteness significantly affects starcounts for $r \lesssim 6^{\prime \prime}$, becoming more and more important toward the center of the cluster, until, as said, it reaches $100 \%$ at $r \lessgtr 2^{\prime \prime}$. However, an excellent match of the profiles can be achieved in the range $10^{\prime \prime} \leq r<20^{\prime \prime}$ (i.e. to the end of the light profile). This clearly proves that for $r \geq 10^{\prime \prime}$ there is no more variation in the incompleteness with radius and, consequently, star counts provide a fair and fully reliable description of the real profile in the considered range. The vertical shift applied to the star-count profile to match the light profile also automatically provides the normalization constant to transform surface stellar densities into surface brightness. Therefore, the two profiles can be joined into one, covering the full $0 \leq r \leq 35^{\prime \prime}$ range, as shown in Fig. 5 .

Is it possible that the excess component beyond $r_{\mathrm{t}}$ and/or the observed change of slope can be due to sources unrelated to the cluster? This possibility is very hard to conceive, since (1) the adopted quality selections (G06b) and the CMD filter limit the analysis to relatively bright, well-behaved stars that should not suffer from any serious contamination, and (2) it is very hard to imagine a "field" population whose surface density decreases with distance from the cluster center. We must conclude that the detected surface density excess at large radius and the change of slope in the profile are genuine properties of the cluster. A change of slope in the outer regions of the

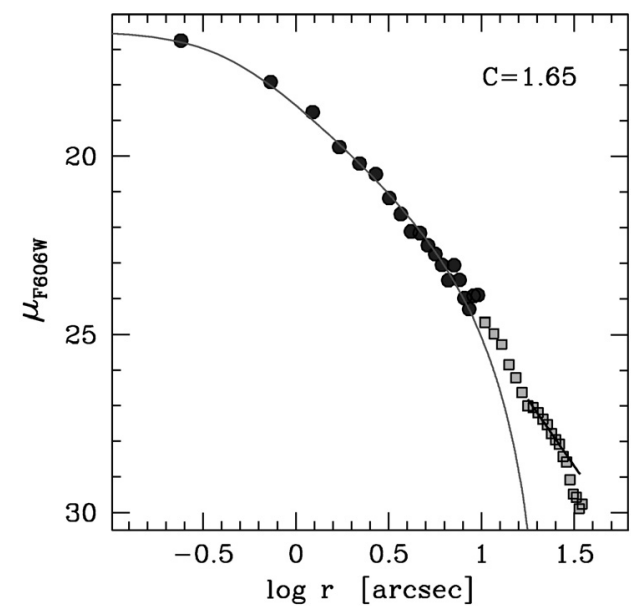

Fig. 5. Total surface brightness profile obtained by joining the light (dark grey circles) and the star-counts (light grey squares) profiles. The light curve is the (PSF-convolved) King model best fitting the light profile. The dark segment in the outer part is a surface density $\propto r^{-3}$ power law.

surface density profile is generally interpreted as the signature of the presence of tidally stripped stars (see Combes et al. 1999; Johnston et al. 1999; Leon et al. 2000, and references therein). For brevity, in the following we will refer to the stars beyond the break in the profile as extra-tidal stars.

\section{Discussion}

A general prediction of theoretical incompleteness studies of tidal tails is that the slope of the surface brightness profile is different for bound stars and extra-tidal stars (see, for example, Combes et al. 1999, C99; Yim \& Lee 2002; Johnston et al. 1999; Montuori et al. 2007). Johnston et al. (1999) predicts that the surface density of stars in the tails should decrease as $r^{-1}$, in agreement with most observations of extra-tidal stars around Galactic globulars (Grillmair et al. 1995; Leon et al. 2000; Testa et al. 2000; Odenkirchen et al. 2003). On the other hand, C99 conclude that such a shallow slope is to be expected in the tidal tails at large distances from the parent cluster - i.e. fully unbound independent tidal debris - while in the vicinity of the cluster i.e. immediately beyond the tidal radius - the density should decrease as $r^{-\alpha}$ with $\alpha=3$ or higher, and the involved stars cannot be considered as completely unbound. C99 explain the discrepancy with the observed slopes as due to imperfect subtraction of a very noisy background, typical in most Galactic cases. It is interesting to note that in the present case, where we deal with stars in the proximity of the cluster and the background is virtually non-existing, the density of extra-tidal component decreases significantly faster than $r^{-1}$, and it is compatible with $\rho_{S} \propto r^{-3}$ (the thick segment superposed to the outer profile in Fig. 5).

In any case, the only possible alternative for explaining the observed excess of stars and the change of slope in the profile at $r_{\mathrm{t}} \simeq 18^{\prime \prime}$ is to postulate that the cluster is embedded in a very low surface-brightness stellar system, such as an unknown dwarf galaxy. We consider this hypothesis as unlikely, because (a) the density of the detected extra-tidal component decreases with distance from the center of the cluster, which would only be possible if the cluster resides at the center of the hypothesized system; (b) the surface brightness of the extra-tidal component is $\gtrsim 26 \mathrm{mag} / \operatorname{arcsec}^{2}$, significantly lower than the typical central surface density of local dwarf spheroidals (Mateo 1998; but see 
Zucker et al. 2004, for a counter-example in M 31); and (c) the INT/WFC survey (Ibata et al. 2001) reaches the surroundings of B514 (the cluster is near one edge of the survey field), and no particular substructure is detected in this region. The twisting and distortion of the outer isophotes noted in Sect. 2.1.1, above, also militates for the tidal origin of the observed structures. The detection of tidal tails on opposite sides with respect to the center of the cluster would have provided a conclusive argument in this sense (see Montuori et al. 2007, and references therein). Unfortunately the extra-tidal over-density only appears as statistically significant when azimuth-averaged. We find just a small degree of anisotropy in the spatial distribution of extratidal stars: they appear to be located preferentially along the SE NW direction, but the effect is not statistically significant.

\subsection{Bright clusters and the nuclei of dwarf ellipticals}

Extragalactic surveys performed with high spatial-resolution cameras are revealing the existence of new kinds of stellar systems. The extended clusters (ECs) found by Huxor (2005) are a typical example, but other kinds of extended clusters have been identified in more distant galaxies (see Peng et al. 2006, for a thorough review and discussion). Hilker et al. (1999) and Drinkwater et al. (2000) have recently discovered a new kind of dwarf galaxies inhabiting galaxy clusters that are slightly more luminous than the brightest globular clusters and significantly more compact than any dwarf galaxy, the ultra compact dwarf galaxies (UCD). It has been suggested that such systems are the dense remnant of tidally harassed nucleated galaxies (Drinkwater et al. 2003). In this context a new perspective on the possible relations and differences among these various system is gradually emerging, and the comparison among the structural properties may provide interesting insights in this sense.

In this line, MB05 compared globular clusters from different galaxies in the $M_{V}$ vs. $\log r_{\mathrm{h}}$ diagnostic plane (see also Hasegan et al. 2005). They found that the bulk of globulars lies below the line $\log r_{\mathrm{h}}=0.25 M_{V}+2.95$. All the objects that are found above this threshold, namely $\omega$ Cen, NGC 2419, M 54, and G1, are very bright and anomalous clusters: all of them were previously indicated as possible remnants of disrupted nucleated dwarf galaxies (Freeman \& Bland-Hawthorn 2002). The $M_{V}$ vs. $\log r_{\mathrm{h}}$ plane has a distinct advantage with respect to other similar diagnostic planes (as, for instance, $M_{V}$ vs. $\mu_{V}(0)$, Kormendy 1985), since $r_{\mathrm{h}}$ is quite an easy-to-measure and reddening independent quantity.

In Fig. 6 we show in the $M_{V}$ vs. $\log r_{\mathrm{h}}$ plane the position of B514 and other interesting systems. Apart of B514, the real novelty of Fig. 6 with respect to previous versions (MB05; Huxor et al. 2005; Belokurov et al. 2007) is that it is now possible to also report the position of the nuclei of dwarf nucleated ellipticals. Côté et al. (2006) measured half-light radii and $g_{A B}, z_{A B}{ }^{3}$ integrated magnitudes of the nuclei of several dwarf ellipticals in Virgo from deep ACS/WFC images. We converted $g_{A B}$ magnitudes into $V$ magnitudes with the transformation

$$
V=g_{A B}-0.31\left(g_{A B}-z_{A B}\right) \quad(\mathrm{rms}=0.1 \mathrm{mag})
$$

that we have obtained from 166 bright stars of the cluster NGC 2419 that are shared between the $g_{A B}, z_{A B}$ photometry we obtained from archive ACS/WFC data and the secondary standards provided by Stetson (2005) for this cluster. Then, we converted the integrated $V$ magnitudes into absolute magnitudes by

${ }^{3} g_{A B}$ and $z_{A B}$ are the ACS/WFC $F 475 W, F 850 L P$ passbands, respectively, calibrated in the ABMAG system; see Côté et al. (2006).

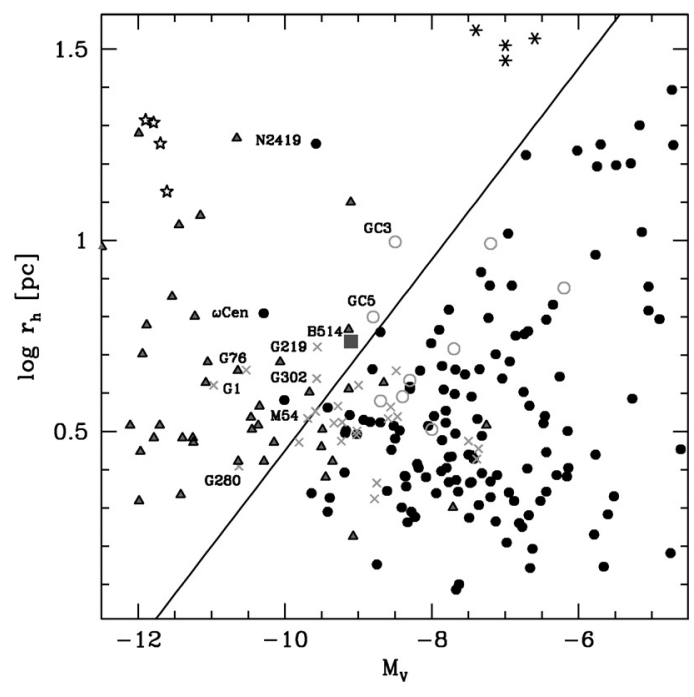

Fig. 6. Absolute integrated $V$ magnitudes vs. logarithm of the half-light radius in pc for various low-luminosity stellar systems. The plotted line is the threshold for ordinary globular clusters in this plane as defined by $\mathrm{MB} 05, \log r_{\mathrm{h}}=0.25 M_{V}+2.95$. Filled circles are Galactic globular clusters, from MB05; grey crosses are M 31 globulars, our own estimates from HST images (these estimates were obtained with a profile analysis strictly homogeneous to that performed here for B514; the results were compared with the independent estimates by BHH: the agreement between the observed parameters from the two sources is very good); asterisks are ECs from Mackey et al. (2006); open circles are outer M 31 GCs from Mackey et al. (2007) (seven of them have $R_{\mathrm{p}}>30 \mathrm{kpc}$ ); open stars are UCDs in the Fornax cluster from De Propris et al. (2005); triangles are nuclei of dwarf elliptical galaxies in the Virgo cluster from Côté et al. (2006); the grey square is B514. The globular clusters lying above the line are labeled with their names.

adopting the reddening and distance modulus provided by Côté et al. (2006).

There are a number of very interesting indications emerging from Fig. 6:

1. In addition to the clusters already noted by MB05, i.e. M 54, $\omega$ Cen, NGC 2419, and G1, there are a few other bright M 31 globulars lying above the "ordinary globular cluster" threshold: B514, M-GC3, M-GC5, G76, G280, G219, and G302. All of these systems are classified as globular clusters but have significantly larger half-light radii than those of typical genuine globular clusters of the same metallicity.

2. The distribution of nuclei overlaps the position of these anomalous clusters nicely. In particular, the nuclei of dwarf ellipticals appear to join the brightest globulars to the anomalous "above-threshold" clusters and to the UCD galaxies, which have been also interpreted as the nuclear remnants of shredded galaxies (Drinkwater et al. 2003). This is the first time that a clear connection between the structure of nuclei, UCDs, and anomalous clusters has been directly established by comparing sizable samples. It would be very interesting to extend the comparison to velocity dispersions, but unfortunately these quantities are not available for the sample of nuclei considered here (but see Hasegan et al. 2005) and for most of M 31 globulars, including B514.

3. ECs, on the other hand, seem to have a different nature, somehow intermediate between globular and open star clusters (Peng et al. 2006).

4. The remote clusters B514 $\left(R_{\mathrm{p}} \simeq 55 \mathrm{kpc}\right)$ and M-GC5 $\left(R_{\mathrm{p}} \simeq\right.$ $78 \mathrm{kpc})$ lie above the $\log r_{\mathrm{h}}-M_{V}$ threshold, while M-GC1 $\left(R_{\mathrm{p}} \simeq 46 \mathrm{kpc}\right)$ and M-GC10 $\left(R_{\mathrm{p}} \simeq 100 \mathrm{kpc}\right)$ are located well 
below the threshold, fully immersed within the distribution of ordinary globular clusters. This demonstrates that a large half-light radius is not a distinctive characteristic of remote clusters.

5. It is interesting to note that the faint Galactic satellites recently discovered by various SDSS teams (see Belokurov et al. 2007, and references therein) lie outside the limits of Fig. 6, all of them having $\log r_{\mathrm{h}}>1.6$ and $M_{V}>-8$, just above ECs. Ordinary dwarf spheroidals have similar sizes but they have $M_{V}<-8$ (Mateo 1998).

It is interesting to have a closer look at the anomalous "abovethreshold" clusters. Both $\omega$ Cen and G1 clearly host stars of different chemical compositions (and, presumably, age), hence unlike classical globulars - they were able to sustain chemical evolution (see, Sollima et al. 2005; Bekki \& Freeman 2003; Meylan et al. 2001, and references therein). Both clusters are quite elliptical in shape, similar to B514. M 54 resides within the nucleus of the Sagittarius dwarf spheroidal (Monaco et al. 2005) and there are indications of a small metallicity spread among its stars (Sarajedini \& Layden 1995). G76 is a relatively metal poor cluster $([\mathrm{Fe} / \mathrm{H}] \simeq-1.3$, Rich et al. 2005 , hereafter $\mathrm{R} 05)$ that is projected onto a very dense star-forming region in the disc of M 31 (Bellazzini et al. 2003). The extreme crowding conditions in this region prevents a clear interpretation of the wide RGB shown in its CMD as obtained by R05, but this feature clearly leaves room for a possible metallicity spread. G280 is a metal-rich $([\mathrm{Fe} / \mathrm{H}] \simeq-0.5, \mathrm{R} 05)$ and bright cluster; like G76 it is projected onto a high-density background. G302 is a metalpoor cluster $([\mathrm{Fe} / \mathrm{H}] \simeq-1.7, \mathrm{R} 05)$ with a blue horizontal branch. Its CMD (R05) is quite clean and it suggests that G302 is a normal single-population cluster. G219 is a metal-poor cluster $([\mathrm{Fe} / \mathrm{H}] \simeq-1.9, \mathrm{R} 05)$ located at $\sim 20 \mathrm{kpc}$ from the center of M 31 . Bellazzini et al. (2003) note that G219 is projected onto the giant stream discovered by Ibata et al. (2001). It is remarkable, in the present context, that both G302 and G219 are among the rare M 31 clusters with a detection of extra-tidal stars, such as B514 (Holland et al. 1997; Grillmair et al. 1996). NGC 2419 is a remote metal-poor Galactic globular whose CMD is very similar to that of B514 (Harris et al. 1997). As B514 it shows no obvious sign of a metallicity spread, but this may be very difficult to find out from the CMD of very metal-poor clusters (see MB05). It has to be noted that NGC 2419 has a half-light radius as large as those of the largest $\mathrm{dE}$ nuclei and UCD galaxies.

In summary, B514 lies fully in the region of the $M_{V}$ vs. $\log r_{\mathrm{h}}$ plane that appears forbidden to ordinary clusters, in the company of a few other clusters, many of which present some kind of peculiarity. The fact that the same region also hosts the nuclei of dwarf ellipticals supports the hypothesis that these bright extended clusters can be the remnant of disrupted galaxies (Freeman \& Bland-Hawthorn 2002; MB05, and references therein; Brodie \& Strader 2006, for a thorough discussion of the role of globular clusters within a cosmological context). On the other hand, it is possible that ordinary clusters are allowed to attain extended structures if their orbit never drives them into the inner part of the parent galaxy, as may be the case of NGC 2419 and B514 (see van den Bergh et al. 1991). However, the coexistence of ordinary and "above-threshold" clusters in the outermost regions of M 31 (see point 4, above) does not support this hypothesis.

The present study confirms that globular clusters in the outskirts of M 31 may reveal many interesting features, thanks also to the favorable observing conditions (as for instance, the very low background). Remote clusters are rare in the Milky Way, and they are typically quite faint, with the only exception of NGC 2419 (see Mackey et al. 2007; and Galleti et al. 2007). Hence, M 31 may provide the opportunity for a systematic study of a kind of stellar system that is very rare in the Galaxy.

Using the same selection criteria that led us to the discovery of B514 (G05), we have selected a conspicuous number of candidate clusters at large distances from the center of M 31. At present we have obtained spectroscopic confirmation (as in G06a) for four of them, all nearly as luminous as B514 (Galleti et al. 2007). The follow up of these remote clusters, as well as of those discovered by other teams, may hopefully open a new window on the study of the M 31 system and of bright clusters as a whole.

Acknowledgements. We are grateful to Dougal Mackey and Avon Huxor for useful discussions. We acknowledge the financial support for this research by Agenzia Spaziale Italiana (ASI) and of the Italian Ministro dell'Università e della Ricerca under the grant INAF/PRIN05 1.06.08.03. Part of the data analysis was carried out with software developed by P. Montegriffo at INAF - Bologna Observatory.

\section{References}

Barmby, P., Holland, S., \& Huchra, J. P. 2002, AJ, 123, 1937 (BHH)

Barmby, P., McLaughlin, D., Harris, W. E., Harris, G. L. H., \& Forbes, D. A. 2007, AJ, 133, 2764

Bates, S. A., Holtzman, J. A., Walterbos, R. A. M., et al. 2004, BAAS, 205, 64.10 Bekki, K., \& Freeman, K. C. 2003, MNRAS, 346, L11

Bellazzini, M., Cacciari, C., Federici, L., Fusi Pecci, F., \& Rich, M. R. 2003, A\&A, 405, 867

Belokurov, V., Evans, N. W., Irwin, M. J., Hewett, P. C., \& Wilkinson, M. I. 2006, ApJ, 637, L29

Belokurov, V., Zucker, D. B., Evans, N. W., et al. 2007, ApJ, 654, 897

Brodie, J. P., \& Strader, J. 2006, ARA\&A, 44, 193

Bullock, J. S., \& Johnston, K. V. 2004, in Satellites and Tidal Streams, ed. F. Prada, D. Martinez-Delgado, \& T. Mahoney (San Francisco: ASP), ASP Conf. Ser., 327, 80

Combes, F., Leon, S., \& Meylan, G. 1999, A\&A, 352, 149 (C99)

Côté, P., Piatek, S., Ferrarese, L., et al. 2006, ApJS, 165, 57

De Propris, R., Phillipps, S., Drinkwater, M. J., et al. 2005, ApJ, 623, L105

Djorgovski, S. G., \& Piotto, G. 1993, in Structure and Dynamics of Globular Clusters, ed. S. G. Djorgovski, \& G. Meylan (San Francisco: ASP), ASP Conf. Ser., 50, 203

Djorgovski, S. G., Piotto, G., Phinney, E. S., \& Chernoff, D. F. 1991, ApJ, 372, L41

Djorgovski, S. G., Côté, P., Meylan, G., et al. 2003, in New Horizons in Globular Cluster Astronomy, ed. G. Piotto, G. Meylan, \& S. G. Djorgovski (San Francisco: ASP), ASP Conf. Ser., 296, 479

Drinkwater, M. J., Jones, J. P., Gregg, M. D., \& Phillipps, S. 2000, PASA, 17, 227

Drinkwater, M. J., Gregg, M. D., Hilker, M., et al. 2003, Nature, 423, 519

Freeman, K. C., \& Bland-Hawthorn, J. 2002, ARA\&A, 40, 487

Fusi Pecci, F., Battistini, P., Bendinelli, O., et al. 1994, A\&A, 284, 349

Galleti, S., Bellazzini, M., Federici, L., \& Fusi Pecci, F. 2005, A\&A, 436, 535 (G05)

Galleti, S., Federici, L., Bellazzini, M., Buzzoni, A., \& Fusi Pecci, F. 2006a, A\&A, 456, 985 (G06a)

Galleti, S., Federici, L., Bellazzini, M., Buzzoni, A., \& Fusi Pecci, F. 2006b, ApJ, 650, L107 (G06b)

Galleti, S., Bellazzini, M., Federici, L., Buzzoni, A., \& Fusi Pecci, F. 2007, A\&A, 471, 127

Grillmair, C., Freeman, K. C., Irwin, M. J., \& Quinn, P. J. 1995, AJ, 109, 2553

Grillmair, C., Ajhar, E. A., Faber, S. M., et al. 1996, AJ, 111, 2293

Johnston, K. V., Sigurdsson, S., \& Hernquist, L. 1999, MNRAS, 302, 771

Harris, W. E. 1996, AJ, 112, 1487

Harris, W. E., Bell, R. A., Vandenberg, D. A., et al. 1997, AJ, 114, 1030

Hasegan, M., Jordàn, A., Côté, P., et al. 2005, ApJ, 627, 203

Hilker, M., Infante, L., Vieira, G., Kissler-Patig, M., \& Richtler, T. 1999, A\&AS, 134,75

Holland, S., Fahlman, G. C., \& Richer, H. B. 1997, AJ, 114, 448

Huxor, A. P., Tanvir, N. R., Irwin, M., et al. 2004, in Satellites and Tidal Streams, ed. F. Prada, D. Martinez-Delgado, \& T. Mahoney (San Francisco: ASP), ASP Conf. Ser., 327, 118

Huxor, A. P., Tanvir, N. R., Irwin, M. J., et al. 2005, MNRAS, 360, 1007 
King, I. R. 1962, AJ, 67, 471

King, I. R. 1966, AJ, 71, 64

Kormendy, J. 1985, ApJ, 295, 73

Ibata, R. A., Irwin, M., Lewis, G., Ferguson, A. M. N., \& Tanvir, N. 2001, Nature, 412,49

Lauer, T. R. 1985, ApJS, 57, 473

Lee, K. H., Lee, H. M., Fahlman, G. G., \& Sung, H. 2004, AJ, 128, 2838

Leon, S., Meylan, G., \& Combes, F. 2000, A\&A, 2000, 359, 907

Mackey, A. D., \& van den Bergh, S. 2005, MNRAS, 360, 631 (MB05)

Mackey, A. D., Huxor, A. P., Ferguson, A. M. N., et al. 2006, ApJ, 653, L105

Mackey, A. D., Huxor, A. P., Ferguson, A. M. N., et al. 2007, ApJ, 655, L88

Martin, N. F., Ibata, R. A., Irwin, M. J., et al. 2006, MNRAS, 371, 1983

Mateo, M. 1998, ARA\&A, 36, 435

McConnachie, A. W., Irwin, M. J., \& Ferguson, A. M. N. 2005, MNRAS, 356, 979

Meylan, G., Sarajedini, A., Jablonka, P., et al. 2001, AJ, 122, 830

Monaco, L., Bellazzini, M., Ferraro, F. R., \& Pancino, P. 2005, MNRAS, 356, 1396
Montuori, M., Capuzzo-Dolcetta, R., Di Matteo, P., Lepinette, A., \& Miocchi, P. 2007, ApJ, 659, 1212

Odenkirchen, M., Grebel, E. K., Dehnen, W., et al. 2003, AJ, 126, 2385

Peng, E. W., Côté, P., Jordàn, A., et al. 2006, ApJ, 639, 838

Rich, R. M., Corsi, C. E., Cacciari, C., et al. 2005, AJ, 129, 2670 (R05)

Sarajedini, A., \& Layden, A. 1985, AJ, 109, 1086

Sirianni, M., Jee, M. J., Benitez, N., et al. 2005, PASP, 117, 1049

Smith, J. A., Tucker, D. L., Kent, S., et al. 2002, AJ, 123, 2121

Sollima, A., Pancino, E., Ferraro, F. R., et al. 2005, ApJ, 634, 332

Spitzer, L. 1987, Dynamical evolution of globular clusters (Princeton: Princeton University Press)

Stetson, P. 2005, PASP, 117, 536

Testa, V., Zaggia, S. R., Andreon, S., et al. 2000, A\&A, 356, 127

van den Bergh, S., Morbey, C., \& Pazder, J. 1991, ApJ, 375, 594

Zucker, D. B., Kniazev, A. Y., Bell, E. F., et al. 2004, ApJ, 612, L121

Yim, K.-J., \& Lee, H. M. 2002, JKAS, 35, 75 
L. Federici et al.: The extended structure of the cluster B514, Online Material $p 1$

\section{Online Material}


L. Federici et al.: The extended structure of the cluster B514, Online Material $p 2$

Table 3. Background-subtracted $F 606 \mathrm{~W}$ surface brightness profile from the integrated light.

\begin{tabular}{ccc}
\hline \hline $\begin{array}{c}r \\
\operatorname{arcsec}\end{array}$ & $\begin{array}{c}\mu_{F 606 W} \\
\mathrm{mag} / \operatorname{arcsec}^{2}\end{array}$ & $\begin{array}{c}\epsilon_{\mu} \\
\mathrm{mag} / \mathrm{arcsec}^{2}\end{array}$ \\
\hline 0.24 & 16.7 & 0.1 \\
0.73 & 17.9 & 0.1 \\
1.23 & 18.8 & 0.2 \\
1.71 & 19.7 & 0.1 \\
2.20 & 20.2 & 0.2 \\
2.69 & 20.5 & 0.2 \\
3.18 & 21.2 & 0.2 \\
3.67 & 21.6 & 0.2 \\
4.16 & 22.1 & 0.2 \\
4.65 & 22.2 & 0.2 \\
5.14 & 22.5 & 0.4 \\
5.63 & 22.8 & 0.2 \\
6.12 & 23.1 & 0.3 \\
6.61 & 23.5 & 0.3 \\
7.11 & 23.1 & 0.6 \\
7.59 & 23.5 & 0.4 \\
8.09 & 24.0 & 0.2 \\
8.57 & 24.3 & 0.4 \\
9.06 & 23.9 & 0.3 \\
9.55 & 23.9 & 0.4 \\
10.05 & 24.1 & 0.4 \\
10.53 & 24.8 & 0.5 \\
11.02 & 24.6 & 0.3 \\
11.51 & 24.8 & 0.3 \\
12.01 & 24.5 & 0.8 \\
12.49 & 25.0 & 0.5 \\
12.98 & 25.4 & 0.4 \\
13.47 & 25.8 & 0.4 \\
13.96 & 26.0 & 0.3 \\
14.45 & 25.7 & 0.4 \\
14.94 & 26.2 & 0.6 \\
15.43 & 26.1 & 0.6 \\
15.92 & 26.2 & 0.6 \\
16.41 & 26.2 & 0.6 \\
16.90 & 26.7 & 0.6 \\
17.40 & 26.7 & 0.7 \\
17.89 & 26.7 & 0.6 \\
18.38 & 26.6 & 1.4 \\
18.86 & 27.0 & 0.5 \\
19.35 & 27.0 & 0.6 \\
\hline & &
\end{tabular}

Table 4. Background-subtracted $F 606 W$ surface brightness profile from star counts.

\begin{tabular}{ccc}
\hline \hline $\begin{array}{c}r \\
\text { arcsec }\end{array}$ & $\begin{array}{c}\mu_{F 606 W}{ }^{a} \\
\mathrm{mag}_{\text {arcsec }}{ }^{2}\end{array}$ & $\begin{array}{c}\epsilon_{\mu} \\
\mathrm{mag} / \mathrm{arcsec}^{2}\end{array}$ \\
\hline 10.41 & 24.7 & 0.1 \\
11.64 & 25.0 & 0.1 \\
12.86 & 25.3 & 0.1 \\
14.09 & 25.8 & 0.1 \\
15.31 & 26.2 & 0.1 \\
16.54 & 26.6 & 0.2 \\
17.76 & 27.0 & 0.2 \\
18.99 & 27.1 & 0.2 \\
20.21 & 27.2 & 0.2 \\
21.44 & 27.4 & 0.2 \\
22.66 & 27.5 & 0.2 \\
23.89 & 27.8 & 0.2 \\
25.11 & 28.0 & 0.2 \\
26.34 & 28.1 & 0.2 \\
27.56 & 28.4 & 0.2 \\
28.79 & 28.6 & 0.2 \\
30.01 & 29.1 & 0.3 \\
31.24 & 29.5 & 0.3 \\
32.46 & 29.6 & 0.3 \\
33.69 & 29.9 & 0.3 \\
34.91 & 29.8 & 0.3 \\
\hline
\end{tabular}

${ }^{a}$ The star counts profile has been converted into surface brightness units $\left(\mathrm{mag} / \operatorname{arcsec}^{2}\right)$ with the relation $\mu_{F 605 W}=-2.5 \log \left(\rho_{S}\right)+23.20$, according to the normalization shown in Fig. 4. 